\title{
Is there a Teichmüller principle in higher dimensions?
}

\author{
Oliver Roth \\ October 6, 2018
}

\begin{abstract}
The underlying theme of Teichmüller's papers in function theory is a general principle which asserts that every extremal problem for univalent functions of one complex variable is connected with an associated quadratic differential. The purpose of this paper is to indicate a possible way of extending Teichmüller's principle to several complex variables. This approach is based on the Loewner differential equation.
\end{abstract}

\section{Introduction}

We denote by $\operatorname{Hol}\left(\mathbb{B}^{n}, \mathbb{C}^{n}\right)$ the set of all holomorphic maps from the open unit ball $\mathbb{B}^{n}:=\left\{z \in \mathbb{C}^{n}:\|z\|<1\right\}$ equipped with the standard Euclidean norm $\|\cdot\|$ of $\mathbb{C}^{n}$ into $\mathbb{C}^{n}$. Endowed with the compact-open topology of locally uniform convergence, the vector space $\mathrm{Hol}\left(\mathbb{B}^{n}, \mathbb{C}^{n}\right)$ becomes a Fréchet space. In geometric function theory, the univalent maps in $\operatorname{Hol}\left(\mathbb{B}^{n}, \mathbb{C}^{n}\right)$ are of particular interest and extremal problems provide an effective method for establishing the existence of univalent maps with certain natural properties.

This point of view is particularly successful in the classical one-dimensional case, since the class

$$
\mathcal{S}:=\left\{f \in \operatorname{Hol}(\mathbb{D}, \mathbb{C}): f(0)=0, f^{\prime}(0)=1, f \text { univalent }\right\}
$$

of all normalized univalent (or schlicht) functions on the unit disk $\mathbb{D}:=\mathbb{B}^{1}$ is a compact subset of $\operatorname{Hol}(\mathbb{D}, \mathbb{C})$ and so any continuous functional $J: S \rightarrow \mathbb{R}$ attains its maximum value within the class $\mathcal{S}$. This means that there exists at least one $F \in \mathcal{S}$ such that $J(f) \leq J(F)$ for any $f \in \mathcal{S}$. We call such a map $F$ an extremal function for $J$ over $S$.

Around 1938, Teichmüller 41] stated a general principle which roughly says that any extremal problem over $\mathcal{S}$ is associated in a well-defined way with a quadratic differential. Teichmüller did not go on to give a precise formulation of his principle in upmost generality, but he did content himself with applying his principle to a number of specific, yet characteristic special cases. Only much later, Jenkins (see

Mathematics Subject Classification (2000) Primary 30C55 - 32H02 - 49K15 
[20]) succeeded in formulating what is called the General Coefficient Theorem and which can be regarded as a rigorous version of Teichmüller's principle for a fairly large class of extremal problems.

In order to state Teichmüller's principle, the following notion is useful.

Definition $1.1([40,25])$

Let $Q$ be a meromorphic function on $\mathbb{C}$. A formal expression of the form

$$
Q(w) d w^{2}
$$

is called a quadratic differential. A function $F \in S$ is called admissible for the quadratic differential $Q(w) d w^{2}$, if $F$ maps $\mathbb{D}$ onto $\mathbb{C}$ minus a set of finitely many analytic $\operatorname{arcs} w=w(t)$ satisfying $Q(w) d w^{2}>0$.

Now, roughly speaking Teichmüller's principle asserts that to each quadratic differential one can associate an extremal problem for univalent functions in such a way that the extremal functions are admissible for this quadratic differential. This principle has a partial converse, first established by Schiffer [36], which says that for any extremal problem for univalent functions one can associate a quadratic differential $Q(w) d w^{2}$ so that the corresponding extremal functions are admissible for $Q(w) d w^{2}$.

For the sake of simplicity, we restrict our discussion to a particular simple, yet important case and consider for a fixed integer $N \geq 2$ the $N$-th coefficient functional

$$
J_{N}(f):=a_{N}, \quad f(z)=z+\sum_{k=2}^{\infty} a_{k} z^{k} \in \operatorname{Hol}(\mathbb{D}, \mathbb{C}) .
$$

For this functional, one can state the Teichmüller-Schiffer paradigma in a precise and simple way, see [8, Chapter 10.8]. We start with the Schiffer differential equation.

Theorem 1.2 (Schiffer's Theorem)

Let $F(z)=z+\sum_{k=2}^{\infty} A_{k} z^{k} \in \mathcal{S}$ be an extremal function for $\operatorname{Re} J_{N}$ over $\mathcal{S}$ and let

$$
P_{N}(w):=\sum_{k=1}^{N-1} J_{N}\left(F^{k+1}\right) w^{k} .
$$

Then $F$ is admissible for the quadratic differential

$$
-P_{N}\left(\frac{1}{w}\right) \frac{d w^{2}}{w^{2}}
$$

and $F$ is a solution to the differential equation

$$
\left[\frac{z F^{\prime}(z)}{F(z)}\right]^{2} P_{N}\left(\frac{1}{F(z)}\right)=R_{N}(z)
$$


with

$$
R_{N}(z)=(N-1) A_{n}+\sum_{k=1}^{N-1}\left(k A_{k} z^{k-N}+k \overline{A_{k}} z^{N-k}\right)
$$

and

$$
R_{N}(\kappa) \geq 0 \quad \text { for all } \kappa \in \partial \mathbb{D} \text { with equality for at least one } \kappa_{0} \in \partial \mathbb{D} \text {. }
$$

Remarks 1.3

A few of remarks are in order.

(a) The only functions $F \in \mathcal{S}$ for which Theorem 1.2 actually applies are the Koebe functions $k(z)=z /(1-\alpha z)^{2}$ with $\alpha^{N-1}=1$. This is a consequence of de Branges' theorem [7], that is, the former Bieberbach conjecture, which states that $\left|a_{N}\right| \leq N$ with equality if and only if $F(z)=z /(1-\eta z)^{2}$ with $|\eta|=1$. However, Schiffer's method works for much more general ("differentiable") functionals $J: \mathcal{S} \rightarrow \mathbb{C}$ as well, see [8]. In this expository paper, we nevertheless focus mainly on the simple functional $J_{N}$ for various reasons. First of all, the more general cases of Schiffer's theorem are essentially as difficult to prove as the case $J_{N}$. Second, the relation to Teichmüller's principle is most easily described for the functional $J_{N}$. Finally, the main issue of this note are extensions to higher dimensions with a view toward a higher dimensional Bieberbach conjecture (see e.g. [5]).

(b) In order to prove Theorem 1.2 one compares the extremal function with nearby functions in the class $S$. The construction of suitable comparison functions is a nontrivial task, because the family $\mathcal{S}$ is highly nonlinear. In one dimension there are several variational methods for univalent functions available. We mention the work of Schiffer, Schaeffer \& Spencer, Goluzin and others, see [8, 25]. These methods are geometric in nature and make use of the Riemann mapping theorem and are thus specifically one dimensional. A different approach is possible by way of the Loewner equation and Pontyagin's Maximum Principle from optimal control. We explain this in more detail below.

(c) Equation (1.2) is called the Schiffer differential equation. It is analogous to the Euler equation in the classical calculus of variations. Like the Euler equation, it expresses the fact that every extremal function is a critical point of $J_{N}$. However, an additional difficulty arises, because the Schiffer differential equation involves the initial coefficients $A_{2}, \ldots, A_{N-1}$ of the unkown extremal function.

(d) It is not difficult to show that $F \in \mathcal{S}$ satisfies Schiffer's equation (1.2) such that the "positivity condition" (1.4) holds if and only if $F$ is admissible for the quadratic differential $-P_{N}(1 / w) \frac{d w^{2}}{w^{2}}$. See the proof of Theorem 1.2 in [8] for the "only if"-part and e.g. [25, Proof of Theorem 7.5] for the "if"-part. 
(e) Theorem 1.2 can further be strengthened by showing that if $F \in \mathcal{S}$ is extremal for the functional $J_{N}$ over $\mathcal{S}$, then $F$ is a one-slit map, that is, $F$ maps $\mathbb{D}$ onto $\mathbb{C}$ minus a single analytic arc. Moreover, this arc has several additional geometric properties such as increasing modulus, the $\pi / 4$-property and an asymptotic direction at infinity, see [8, Chapter 10] for more on this.

Theorem 1.4 (Teichmüller's Coefficient Theorem [41])

Let $P(w)=w^{N-1}+c_{N-2} w^{N-2}+\cdots+c_{1} w+c_{0}$ be a polynomial. Suppose that

$$
F(z)=z+\sum_{k=2}^{\infty} A_{k} z^{k} \in \mathcal{S}
$$

is admissible for the quadratic differential

$$
-P\left(\frac{1}{w}\right) \frac{d w^{2}}{w^{2}}
$$

Then

$$
\operatorname{Re} J_{N}(f) \leq \operatorname{Re} J_{N}(F)
$$

for any

$$
f \in \mathcal{S}\left(A_{2}, \ldots, A_{N-1}\right):=\left\{f(z)=z+\sum_{k=2}^{\infty} a_{k} z^{k} \in \mathcal{S}: a_{2}=A_{2}, \ldots, a_{N-1}=A_{N-1}\right\} .
$$

Equality occurs only for $f=F$.

In short, under the assumptions of Theorem 1.4, that is, if $F \in \mathcal{S}$ is a solution to the Schiffer differential equation (1.2) such that the positivity condition (1.4) holds, then $F$ is an extremal function for the real part of the Bieberbach functional $J_{N}(f)$, but subject to the side conditions $a_{2}=A_{2}, \ldots, a_{N-1}=A_{N-1}$ :

\section{Conclusion 1.5}

Let $F(z)=z+\sum_{k=2}^{\infty} A_{k} z^{k} \in \mathcal{S}$. Then the condition that

$F$ is a solution to Schiffer's differential equation (1.2) such that (1.4) holds

is

(a) necessary for $F$ being extremal for $\operatorname{Re} J_{N}$ over the entire class $\mathcal{S}$, and

(b) sufficient for $F$ being extremal for $\operatorname{Re} J_{N}$ over the restricted class $\mathcal{S}\left(A_{2}, \ldots, A_{N-1}\right)$.

Remark 1.6

Teichmüller was quite confident about his result and he conjectured 1 that it can be used to solve the

\footnotetext{
1 "Ich vermute, die Gesamtheit dieser Ungleichungen liefere eine vollständige Lösung des Bieberbachschen Koeffizientenproblems" [41, p. 363]
} 


\section{General Coefficient Problem for Univalent Functions}

Given $F(z)=z+\sum_{k=2}^{\infty} A_{k} z^{k} \in \mathcal{S}$. Find for each $N \in \mathbb{N}$,

$$
\left\{a_{N}: f(z)=z+\sum_{k=2}^{\infty} a_{k} z^{k} \in \mathcal{S}\left(A_{2}, \ldots, A_{N-1}\right)\right\}
$$

The goal of this note is to show that Schiffer's theorem can be extended, at least in spirit, to higher dimensions using the Loewner equation. We also give a statement of Teichmüller's Coefficient Theorem entirely in terms of the Loewner equation. This principally opens up the possibility for an extension of Teichmüller's principle to higher dimensions.

The literature on univalent functions in general, and the Loewner equation in particular, is extensive and there are several excellent survey papers available, see, e.g. [3]. We therefore have included only few references about the subject. As this paper is expository, it contains virtually no proof. An exception is Theorem 6.1.

\section{The Loewner differential equation and the class $S_{n}^{0}$.}

In higher dimensions, a major issue is the fact that the class

$$
\mathcal{S}_{n}:=\left\{f \in \operatorname{Hol}\left(\mathbb{B}^{n}, \mathbb{C}^{n}\right): f(0)=0, D f(0)=\mathrm{id}, f \text { univalent }\right\}
$$

of all normalized univalent mappings on $\mathbb{B}^{n}$ is not compact for any $n \geq 2$. This is easily seen e.g. by considering the noncompact family of shear mappings

$$
z=\left(z_{1}, \ldots, z_{n}\right) \mapsto\left(z_{1}+\alpha z_{2}^{2}, z_{2}, \ldots, z_{n}\right), \quad \alpha \in \mathbb{C}
$$

which all belong to $\mathcal{S}_{n}$. In particular, continuous functionals $J: \mathcal{S}_{n} \rightarrow \mathbb{R}$ do not even need to have upper bounds if $n \geq 2$. In order to study extremal problems for univalent functions in higher dimensions, it is therefore necessary to single out a compact subclass of $\mathcal{S}_{n}$, and one of the most studied classes in this connection is the class $S_{n}^{0}$ of all mappings that admit a so-called parametric representation by means of the Loewner differential equation. It turns out that the classes $S_{n}^{0}$ are compact for each $n \geq 2$ and that $\mathcal{S}_{1}^{0}=\mathcal{S}$.

We now briefly describe the classes $\mathcal{S}_{n}^{0}$ using almost standard notation.

\section{Definition 2.1}

Let

$$
\mathbb{U}_{n}:=\left\{h \in \operatorname{Hol}\left(\mathbb{B}^{n}, \mathbb{C}^{n}\right): h(0)=0, D h(0)=-\mathrm{id}, \operatorname{Re}\langle h(z), z\rangle \leq 0 \text { for all } z \in \mathbb{B}^{n}\right\} .
$$

Here, $\langle\cdot, \cdot\rangle$ denotes the canonical Euclidean inner product of $\mathbb{C}^{n}$. 
It is not difficult to see that a mapping $h \in \operatorname{Hol}\left(\mathbb{B}^{n}, \mathbb{C}^{n}\right)$ satisfying $h(0)=0$ and $D h(0)=-$ id belongs to $\mathbb{U}_{n}$ if and only if $\operatorname{Re}\langle-h(z), z\rangle>0$ for all $z \in \mathbb{B}^{n} \backslash\{0\}$, see [4, Remark 2.1]. In particular, the set $\mathbb{U}_{n}$ is exactly the class $-\mathcal{M}$ with $\mathcal{M}$ as defined e.g. in [16, p. 203].

Theorem 2.2 ([16]: Theorem 6.1.39)

$\mathbb{U}_{n}$ is a compact and convex subset of $\operatorname{Hol}\left(\mathbb{B}^{n}, \mathbb{C}^{n}\right)$.

\section{Definition 2.3}

Let $\mathbb{R}_{0}^{+}:=\{t \in \mathbb{R}: t \geq 0\}$. A Herglotz vector field in the class $\mathbb{U}_{n}$ is a mapping $G: \mathbb{B}^{n} \times \mathbb{R}_{0}^{+} \rightarrow \mathbb{C}^{n}$ such that

(i) $G(z, \cdot)$ is measurable on $\mathbb{R}_{0}^{+}$for every $z \in \mathbb{B}^{n}$, and

(ii) $G(\cdot, t) \in \mathbb{U}_{n}$ for a.e. $t \in \mathbb{R}_{0}^{+}$.

Theorem 2.4 (The Loewner Equation)

Let $G$ be a Herglotz vector field in the class $\mathbb{U}_{n}$. Then for any $z \in \mathbb{B}^{n}$ there is a unique solution $\mathbb{R}_{0}^{+} \ni t \mapsto \varphi(t, z) \in \mathbb{B}^{n}$ of the initial value problem

$$
\begin{aligned}
\frac{\partial \varphi}{\partial t}(t, z) & =G(\varphi(t, z), t) \quad \text { for a.e. } t \geq 0 \\
\varphi(0, z) & =z
\end{aligned}
$$

For each $t \geq 0$, the mapping $e^{t} \varphi(t, \cdot): \mathbb{B}^{n} \rightarrow \mathbb{C}$ belongs to $\mathcal{S}_{n}$ and the limit

$$
f^{G}:=\lim _{t \rightarrow \infty} e^{t} \varphi(t, \cdot)
$$

exists locally uniformly in $\mathbb{B}^{n}$ and belongs to $\mathcal{S}_{n}$.

We refer to [16, Thm. 8.1.5] for the proof. The differential equation in (2.1) is the Loewner equation (in $\mathbb{C}^{n}$ ). It induces a map from the set of all Herglotz vector fields in the class $\mathbb{U}_{n}$ into the set $\mathcal{S}_{n}$. The range of this map plays a crucial role in this paper:

\section{Theorem 2.5}

The set

$$
\mathcal{S}_{n}^{0}:=\left\{f^{G} \in \operatorname{Hol}\left(\mathbb{B}^{n}, \mathbb{C}^{n}\right) \mid G \text { Herglotz vector field in the class } \mathbb{U}_{n}\right\}
$$

is a compact subset of $\operatorname{Hol}\left(\mathbb{B}^{n}, \mathbb{C}^{n}\right)$ for each $n \in \mathbb{N}$, and $\mathcal{S}_{1}^{0}=\mathcal{S}$.

We refer to [16, Corollary 8.3.11] for a proof of the first statement. The fundamental fact that $S_{1}^{0}=S$ is a result of Pommerenke [24, see also [25, Chapter 6.1]. We also note that $e^{t} \varphi(t, \cdot) \in S_{n}^{0}$ for all $t \geq 0$ for every solution to (2.1), see e.g. [39, Lemma 2.6]. The class $\mathcal{S}_{n}^{0}$ is exactly the class of mappings in $\operatorname{Hol}\left(\mathbb{B}^{n}, \mathbb{C}^{n}\right)$ which 
have a parametric representation as introduced by Graham, Hamada and Kohr [12, Definition 1.5], see also [13, 16].

Since the class $S_{n}^{0}$ is compact, we can ask for sharp coefficient bounds as in the one-dimensional case. More precisely, let $f \in \mathcal{S}_{n}^{0}$. We write $f=\left(f_{1}, \ldots, f_{n}\right)$ with $f_{j} \in \operatorname{Hol}\left(\mathbb{B}^{n}, \mathbb{C}\right)$, and consider the coefficient functionals

$$
J_{\alpha}(f):=a_{\alpha}, \quad f_{1}(z)=z_{1}+\sum_{\alpha \in \mathbb{N}_{0}^{n},|\alpha| \geq 2} a_{\alpha} z^{\alpha}
$$

Here $\alpha=\left(\alpha_{1}, \ldots, \alpha_{n}\right) \in \mathbb{N}_{0}^{n}$ denotes a multi-index and $|\alpha|=\alpha_{1}+\ldots+\alpha_{n}$. In view of the Bieberbach conjecture, it is natural to consider the extremal problem

$$
\max _{f \in \mathcal{S}_{n}^{0}} \operatorname{Re} J_{\alpha}(f)
$$

for each multi-index $\alpha \in \mathbb{N}_{0}^{N}$ such that $|\alpha| \geq 2$. As in the one-dimensional case, we call a mapping $F \in \mathcal{S}_{n}^{0}$ extremal for the functional $\operatorname{Re} J_{\alpha}$ over $\mathcal{S}_{n}^{0}$, if

$$
\operatorname{Re} J_{\alpha}(F)=\max _{f \in \mathcal{S}_{n}^{0}} \operatorname{Re} J_{\alpha}(f)
$$

We can now formulate the problem.

\section{Problem 2.6}

For a multi-index $\alpha \in \mathbb{N}_{0}^{N}$ with $|\alpha| \geq 2$ find a necessary condition for an extremal mapping $F \in \mathcal{S}_{n}^{0}$ for the functional $\operatorname{Re} J_{\alpha}$ over $\mathcal{S}_{n}^{0}$. For the special case $n=1$ this condition should reduce to the Schiffer differential equation (1.2). In addition, this necessary condition should be a sufficient condition for extremality under suitable side conditions.

\section{Control-theoretic interpretation of the Loewner equation}

In this section we give an interpretation of the Loewner equation as an infinitedimensional control system in the Fréchet space $\operatorname{Hol}\left(\mathbb{B}^{n}, \mathbb{C}^{n}\right)$. For this purpose, we gradually begin to change notation.

\section{Remark 3.1}

It seems that Loewner himself was the first who came up with the idea of applying methods from optimal control theory to the Loewner equation. In 1967, his last student G.S. Goodman [11] combined Loewner's theory with the then new Pontryagin Maximum Principle. Since then this approach has been used by many others, see e.g. [26, 1, 9, 10] and in particular the important contributions of D.V. Prokhorov [27, 29, 30, 31]. Recent applications of optimal control methods to univalent functions can be found e.g. in [28, 22]. 
Definition 3.2 (Admissible controls, control set)

Let $G$ be a Herglotz vector field in the class $\mathbb{U}_{n}$. We call the mapping

$$
u=u_{G}: \mathbb{R}_{0}^{+} \rightarrow \mathbb{U}_{n}, \quad t \mapsto G(\cdot, t),
$$

an admissible control (for the Loewner equation) and denote by $\mathcal{U}_{n}$ the collection of all admissible controls. The class $\mathbb{U}_{n}$ is called the control set (of the Loewner equation).

Remark 3.3 (Herglotz vector fields as $\operatorname{Hol}\left(\mathbb{B}^{n}, \mathbb{C}^{n}\right)$-valued measurable controls)

One might think of an admissible control as a measurable mapping defined on the time interval $\mathbb{R}_{0}^{+}=[0, \infty)$ and with values in the control set $\mathbb{U}_{n}$, which is a subset of the infinite dimensional Fréchet space $\operatorname{Hol}\left(\mathbb{B}^{n}, \mathbb{C}^{n}\right)$.

We continue to change notation and denote in the sequel the solutions $\varphi(t, \cdot)$ of the Loewner equation (2.1) by $\varphi_{t}$. Note that $\varphi_{t}$ always belongs to the composition semigroup

$$
\mathbb{S}_{n}:=\left\{\varphi \in \operatorname{Hol}\left(\mathbb{B}^{n}, \mathbb{C}^{n}\right): \varphi\left(\mathbb{B}^{n}\right) \subseteq \mathbb{B}^{n}, \varphi(0)=0\right\}
$$

of all holomorphic selfmaps of the unit ball $\mathbb{B}^{n}$.

Definition 3.4 (Trajectories, state space)

Let $u \in \mathcal{U}_{n}$ be an admissible control. Denote by $G$ the Herglotz vector field in the class $\mathbb{U}_{n}$ such that $u=u_{G}$ and let $t \mapsto \varphi_{t}$ be the solution of the Loewner equation (2.1) corresponding to $G$. Then we call the curve

$$
x:=x_{u}: \mathbb{R}_{0}^{+} \rightarrow \mathbb{S}_{n}, \quad t \mapsto x_{u}(t):=\varphi_{t},
$$

the trajectory (of the Loewner equation) for $u$. The set $\mathbb{S}_{n}$ is called the state space (of the Loewner equation).

Remark 3.5 (Solutions of the Loewner equation as $\mathbb{S}_{n}$-valued curves)

One might think of an trajectory $x: \mathbb{R}_{0}^{+} \rightarrow \mathbb{S}_{n}$ of the Loewner equation as an a.e. differentiable (and absolutely continuous) curve defined on the time interval $\mathbb{R}_{0}^{+}=[0, \infty)$ and with values in the state space $\mathbb{S}_{n}$. The initial point of the curve is the identity map.

In fact, this remark needs clarification. If $x=x_{u}: \mathbb{R}_{0}^{+} \rightarrow \mathbb{S}_{n}$ is a trajectory of the Loewner equation for $u=u_{G}$, then for each $z \in \mathbb{B}^{n}$ there is set $N_{z} \subseteq \mathbb{R}_{0}^{+}$of measure zero such that the solution $t \mapsto \varphi(t, z)$ of (2.1) is differentiable on $\mathbb{R}_{0}^{+} \backslash N_{z}$ and the Loewner equation holds for each $t \in \mathbb{R}_{0}^{+} \backslash N_{z}$. A normal family argument shows that there is in fact a set $N \subseteq \mathbb{R}_{0}^{+}$of measure zero, which does not depend on $z$, such that the Loewner equation (2.1) holds for each $t \in \mathbb{R}_{0}^{+} \backslash N$ and each $z \in \mathbb{B}^{n}$. In addition,

$$
\grave{x}(t):=\frac{\partial \varphi_{t}}{\partial t} \in \operatorname{Hol}\left(\mathbb{B}^{n}, \mathbb{C}^{n}\right) \quad \text { for every } t \in \mathbb{R}_{0}^{+} \backslash N .
$$


Note that

$$
\dot{x}(t)=\frac{\partial \varphi}{\partial t}(t, \cdot)=G(\varphi(t, \cdot), t)=u(t) \circ x(t), \quad t \in \mathbb{R}_{0}^{+} \backslash N
$$

Conclusion 3.6 (The Loewner equation as a control system on $\operatorname{Hol}\left(\mathbb{B}^{n}, \mathbb{C}^{n}\right)$ ) The Loewner equation has the following embarassingly simple form

$$
\begin{aligned}
\dot{x} & =u \circ x, \\
x(0) & =\mathrm{id} .
\end{aligned}
$$

This is a control system on the infinite dimensional Fréchet space $\operatorname{Hol}\left(\mathbb{B}^{n}, \mathbb{C}^{n}\right)$ with state space $\mathbb{S}_{n}$ and control set $\mathbb{U}_{n}$.

\section{Remark 3.7}

The simple form (3.1) of the Loewner equation is due to E. Schippers [38, Proposition 6].

\section{Remark 3.8}

If we define

$$
g: \mathbb{S}_{n} \times \mathbb{U}_{n} \rightarrow \operatorname{Hol}\left(\mathbb{B}^{n}, \mathbb{C}^{n}\right), \quad g(x, u):=u \circ x,
$$

then the Loewner equation (3.1) takes the "traditional" form of a control system:

$$
\begin{aligned}
& \dot{x}(t)=g(x(t), u(t)) \quad \text { for a.e. } t \geq 0, \\
& x(0)=\mathrm{id} .
\end{aligned}
$$

Note that the Loewner equation (3.1) is a control system which is linear with respect to the control.

We can now consider the reachable set for time $t$ of the Loewner equation, that is, the set $\left\{x_{u}(t): u \in \mathcal{U}_{n}\right\}$. However, in view of $e^{t} x_{u}(t) \in \mathcal{S}_{n}^{0}$ for each $t \geq 0$ and each $u \in \mathcal{U}_{n}$ (see Theorem 2.5), we strongly prefer to slightly abuse language and call

$$
\mathcal{R}(t):=\left\{e^{t} x_{u}(t): u \in \mathcal{U}_{n}\right\}, \quad t \in \mathbb{R}_{0}^{+}, \quad \text { resp. } \quad \mathcal{R}(\infty):=\left\{\lim _{t \rightarrow \infty} e^{t} x_{u}(t): u \in \mathcal{U}_{n}\right\}
$$

the reachable set for time $t \in \mathbb{R}_{+}^{0} \cup\{\infty\}$ and

$$
\mathcal{R}:=\bigcup_{t \in[0, \infty]} \mathcal{R}(t)
$$

the overall reachable set of the Loewner equation.

Using these notions, our considerations can be summarized as follows.

Theorem 3.9 ( $S_{n}^{0}=$ reachable set of the Loewner equation)

$$
S_{n}^{0}=\mathcal{R}=\mathcal{R}(\infty)
$$


Remark 3.10

The idea of viewing the classes $\mathcal{S}_{n}^{0}$ as reachable sets of the Loewner equation has been pioneered by Prokhorov [29, 30, 31] for $n=1$ and by Graham, Hamada, G. Kohr and M. Kohr [14, 15] for $n>1$.

With the help of Theorem 3.9, extremal problems over the class $S_{n}^{0}$ can be treated as optimal control problems. For the sake of simplicity, we again consider only extremal problems involving Taylor coefficients of univalent maps.

\section{Definition 3.11}

Let $\alpha \in \mathbb{N}_{0}^{N}$ be a multi-index with $|\alpha| \geq 2$. An admissible control $u^{*} \in \mathcal{U}_{n}$ is called an optimal control for the functional $J_{\alpha}: \operatorname{Hol}\left(\mathbb{B}^{n}, \mathbb{C}^{n}\right) \rightarrow \mathbb{C}$ on $\mathcal{S}_{n}^{0}$ if the univalent mapping

$$
F_{u^{*}}:=\lim _{t \rightarrow \infty} e^{t} x_{u^{*}}(t) \in \mathcal{S}_{n}^{0}
$$

is extremal for $\operatorname{Re} J_{\alpha}$ over $S_{n}^{0}$.

Note carefully, that if $F \in \mathcal{S}_{n}^{0}$ is extremal for $\operatorname{Re} J_{\alpha}$ over $\mathcal{S}_{n}^{0}$, then any admissible control $u:[0, \infty) \rightarrow \mathbb{U}_{n}$ which generates $F$ in the sense that $F=F_{u}$, is an optimal control for $J_{\alpha}$. Problem 2.6 can now be stated in control theoretic terms.

\section{Problem 3.12}

Let $\alpha \in \mathbb{N}_{0}^{N}$ be a multi-index with $|\alpha| \geq 2$. Find a necessary condition for an optimal control for the functional $J_{\alpha}: \operatorname{Hol}\left(\mathbb{B}^{n}, \mathbb{C}^{n}\right) \rightarrow \mathbb{C}$. In addition, this necessary condition should be a sufficient condition for extremality under suitable side conditions.

\section{The Pontryagin Maximum Principle for the class $\mathcal{S}_{n}^{0}$}

The standard necessary condition for an optimal control is provided by the

$$
\text { Pontryagin Maximum Principle, }
$$

see [2]. We first consider the standard finite dimensional version.

Remark 4.1 (Prelude to the Pontryagin Maximum Principle)

Let $V$ be a finite dimensional complex vector space. Suppose that the "state space" $S$ is an open subset of $V$ and that the "control set" $U$ is a compact subset of $V$. Let $g: S \times U \rightarrow V$ be a $C^{1}$-function and let $x_{0} \in S$ be a fixed "initial" state.

(I) The control system

Consider

$$
\begin{aligned}
\dot{x}(t) & =g(x(t), u(t)) \quad \text { for a.e. } t \geq 0, \\
x(0) & =x_{0},
\end{aligned}
$$

and call a measurable control $u: \mathbb{R}_{0}^{+} \rightarrow U$ admissible (for the system (4.1)), if the solution $x=x_{u}$ to (4.1) exists for all a.e. $t \in \mathbb{R}_{0}^{+}$. 
(II) The optimal control problem

Let $J \in V^{*}$, that is, let $J: V \rightarrow \mathbb{C}$ be a $\mathbb{C}$-linear (continuous) functional and let $T>0$ be fixed. We call an admissible control $u^{*}: \mathbb{R}_{0}^{+} \rightarrow U$ an optimal control for the functional $J: V \rightarrow \mathbb{C}$ on $S$ and time $T>0$, if

$$
\operatorname{Re} J\left(x_{u^{*}}(T)\right)=\max \left\{\operatorname{Re} J\left(x_{u}(T)\right): u: \mathbb{R}_{0}^{+} \rightarrow U \text { admissible }\right\} .
$$

(III) The adjoint equation

Suppose that $u: \mathbb{R}_{0}^{+} \rightarrow U$ is an admissible control and $x=x_{u}: \mathbb{R}_{0}^{+} \rightarrow S$ is the solution to (4.1). Then the linear (matrix) equation

$$
\begin{aligned}
\dot{\Phi}(t) & =-\Phi(t) D_{x} g(x(t), u(t)) \\
\Phi(T) & =\mathrm{id}
\end{aligned}
$$

is called the adjoint equation along $(x, u)$ at $T$. Note that $\Phi(t) \in \mathcal{L}(V)$, the set of linear (continuous) endomorphisms of $V$.

(IV) The Hamiltonian

The function $\mathcal{H}: S \times \mathcal{L}(V) \times U \rightarrow \mathbb{C}$ defined by

$$
\mathcal{H}(x, \Phi, u):=J(\Phi \cdot g(x, u))
$$

is called the complex Hamiltonian for the extremal problem (4.2). Note that if we denote the transpose of a map $\Phi \in \mathcal{L}(V)$ by $\Phi_{*}$, then

$$
\mathcal{H}(x, \Phi, u)=\Phi_{*}(J) g(x, u)
$$

We can now state the Pontryagin Maximum Principle:

Theorem 4.2 (Pontryagin Maximum Principle)

Let $V$ be a finite dimensional complex vector space and $J \in V^{*}$. Suppose that $u^{*}: \mathbb{R}_{0}^{+} \rightarrow U$ is an optimal control for $J: V \rightarrow \mathbb{C}$ on $S$ and time $T>0$. Denote by $\Phi^{*}: \mathbb{R}_{0}^{+} \rightarrow \mathcal{L}(V)$ the solution to the adjoint equation (4.3) along $\left(x_{u^{*}}, u^{*}\right)$ at $T$, then

$\operatorname{Re} \mathcal{H}\left(x_{u^{*}}(t), \Phi^{*}(t), u^{*}(t)\right)=\max _{u \in U} \operatorname{Re} \mathcal{H}\left(x_{u^{*}}(t), \Phi^{*}(t), u\right) \quad$ for a.e. $t \in[0, T]$.

The conclusion of Theorem 4.2 means that for a.e. $t \in \mathbb{R}_{0}^{+}$the value $u^{*}(t)$ of the optimal control $u^{*}$ provides a maximum for the function

$$
u \mapsto \operatorname{Re} \Phi^{*}(t)_{*}(J) g\left(x_{*}(t), u\right)
$$

over the control set $U$. For a proof of Theorem 4.2 we refer to any textbook on optimal control theory, see e.g. [43, p. $152 \mathrm{ff}$.]. 
Remark 4.3 (On the definition of the Hamiltonian/The costate equation)

Our definition of the Hamiltonian is slightly nonstandard. However, it is easy to see its relation to the standard Hamiltonian formalism. Note that for $V=\mathbb{C}^{n}$ we can identify the dual space $V^{*}$ with $V$ and we can write the $\mathbb{C}$-linear functional $J: V \rightarrow \mathbb{C}$ as $J(y)=\bar{\eta}^{T} y$ for some vector $\eta \in V$. Hence, if $\Phi: \mathbb{R}_{0}^{+} \rightarrow \mathcal{L}(V)$ is the solution of the adjoint equation (4.3) along $(x, u)$ at $T$, then

$$
\Psi:=\bar{\eta}^{T} \Phi=J(\Phi \cdot)=\Phi_{*}(J): \mathbb{R}_{+}^{0} \rightarrow V^{*}
$$

is the solution to the so-called costate equation

$$
\begin{aligned}
\dot{\Psi}(t) & =-\Psi(t) D_{x} g(x(t), u(t)) \\
\Psi(T) & =\bar{\eta}^{T}
\end{aligned}
$$

Therefore, the standard complex Hamiltonian for the extremal problem (4.2),

$$
H: S \times V^{*} \times U \rightarrow \mathbb{C}, \quad H(x, \Psi, u):=\Psi^{T} g(x, u),
$$

see [21, 30], is related to (our) complex Hamiltonian by

$$
\mathcal{H}(x, \Phi, u)=H(x, \Psi, u), \quad \Psi=\bar{\eta}^{T} \Phi \text {. }
$$

We next apply the Pontryagin machinery as outlined above to the abstract Loewner equation (3.1), so we replace the finite dimensional complex vector space $V$, the state space $S \subseteq V$, the control set $U \subseteq V$ and the control system $g: S \times U \rightarrow V$ by

$$
V=\operatorname{Hol}\left(\mathbb{B}^{n}, \mathbb{C}^{n}\right), \quad S=\mathbb{S}_{n}, \quad U=\mathbb{U}_{n}, \quad g(x, u)=x \circ u .
$$

\section{Remark 4.4}

As we shall see, our sligthly nonstandard definition of the Hamiltonian proves itself as user-friendly. The main reason for this is that the dual $\operatorname{Hol}\left(\mathbb{B}^{n}, \mathbb{C}^{n}\right)^{*}$ of $\operatorname{Hol}\left(\mathbb{B}^{n}, \mathbb{C}^{n}\right)$ can no longer be identified with $\operatorname{Hol}\left(\mathbb{B}^{n}, \mathbb{C}^{n}\right)$, and in fact the topological structure of $\operatorname{Hol}\left(\mathbb{B}^{n}, \mathbb{C}^{n}\right)^{*}$ is fairly complicated, see [6, [42, 17, 18]. However, it will turn out that in case of the Loewner equation, the solutions of the associated adjoint equation actually live in the "decent" vector space $\operatorname{Hol}\left(\mathbb{B}^{n}, \mathbb{C}^{n \times n}\right)$ of all holomorphic maps from $\mathbb{B}^{n}$ into $\mathbb{C}^{n \times n}$. Note that each $A \in \operatorname{Hol}\left(\mathbb{B}^{n}, \mathbb{C}^{n \times n}\right)$ gives rise to the continuous linear operator $\mathcal{A} \in \mathcal{L}\left(\operatorname{Hol}\left(\mathbb{B}^{n}, \mathbb{C}^{n}\right)\right)$ defined by

$$
\mathcal{A} f:=A(\cdot) f, \quad f \in \operatorname{Hol}\left(\mathbb{B}^{n}, \mathbb{C}^{n}\right) .
$$

We proceed in a purely formal way in order to emphasize the analogy with the finite dimensional case, but wish to point out that the following formal considerations can be made rigorous using the elegant Fréchet space calculus developed by R. Hamilton [19]. We start with the adjoint equation for the Loewner ODE. 


\begin{tabular}{|l||c||c|}
\hline & Control System on $\mathbb{C}^{n}$ & Loewner Equation \\
\hline \hline State space & $S \subseteq \mathbb{C}^{n}$ & $\mathbb{S}_{n} \subseteq \operatorname{Hol}\left(\mathbb{B}^{n}, \mathbb{C}^{n}\right)$ \\
\hline Control set & $U \subseteq \mathbb{C}^{n}$ & $\mathbb{U}_{n} \subseteq \operatorname{Hol}\left(\mathbb{B}^{n}, \mathbb{C}^{n}\right)$ \\
\hline Controls & $u: \mathbb{R}_{0}^{+} \rightarrow U$ & $u: \mathbb{R}_{0}^{+} \rightarrow \mathbb{U}_{n}$ \\
\hline Trajectories & $x: \mathbb{R}_{0}^{+} \rightarrow S$ & $x: \mathbb{R}_{0}^{+} \rightarrow \mathbb{S}_{n}$ \\
\hline Equation & $\dot{x}=g(x, u)$ & $\dot{x}=u \circ x$ \\
\hline
\end{tabular}

Table 1: Loewner equation as a control system on $\operatorname{Hol}\left(\mathbb{B}^{n}, \mathbb{C}^{n}\right)$

Remark 4.5 (The adjoint equation of the Loewner ODE)

Recall the Loewner ODE in abstract form

$$
\dot{x}=u \circ x \text {. }
$$

Taking the "functional" derivative of the mapping on the right-hand side,

$$
g: \mathbb{S}_{n} \times \mathbb{U}_{n} \rightarrow \operatorname{Hol}\left(\mathbb{B}^{n}, \mathbb{C}^{n}\right), \quad g(x, u):=u \circ x,
$$

with respect to $x \in \mathbb{S}_{n}$, we get

$$
\frac{\partial(u \circ x)}{\partial x}=D u \circ x
$$

Here, $D u \in \operatorname{Hol}\left(\mathbb{B}^{n}, \mathbb{C}^{n \times n}\right)$ is the total derivative of $u \in \operatorname{Hol}\left(\mathbb{B}^{n}, \mathbb{C}^{n}\right)$ and hence $D u \circ x \in \operatorname{Hol}\left(\mathbb{B}^{n}, \mathbb{C}^{n}\right)$. Therefore, the adjoint equation of the Loewner ODE is

$$
\begin{aligned}
\dot{\Phi}(t) & =-\Phi(t) \cdot D u(t) \circ x(t) \\
\Phi(T) & =\mathrm{id} .
\end{aligned}
$$

Recall that for each $t \in \mathbb{R}_{0}^{+}$the map $\Phi(t)$ is a continuous linear operator on $\operatorname{Hol}\left(\mathbb{B}^{n}, \mathbb{C}^{n}\right)$. It is now easy to find the solution of (4.6) in terms of $D x$. In fact, just take the total derivative of $\dot{x}=u \circ x \in \operatorname{Hol}\left(\mathbb{B}^{n}, \mathbb{C}^{n}\right)$ and get

$$
\dot{D} x=D u \circ x \cdot D x \text {. }
$$

Hence using $D x \cdot(D x)^{-1}=\mathrm{id}$, we see that

$$
(D \dot{x})^{-1}=-(D x)^{-1} D u \circ x .
$$


This means that the solution to the adjoint equation (4.6) along $(x, u)$ at $T$ is simply

$$
t \mapsto \Phi(t)=D x(T) \cdot(D x(t))^{-1} \in \operatorname{Hol}\left(\mathbb{B}^{n}, \mathbb{C}^{n \times n}\right) \subseteq \mathcal{L}\left(\operatorname{Hol}\left(\mathbb{B}^{n}, \mathbb{C}^{n}\right)\right)
$$

and the corresponding Hamiltonian of the Loewner equation (3.1) for the extremal problem (4.2) has the form

$$
\mathcal{H}(x, \Phi, u)=J\left(D x(T) \cdot(D x)^{-1} \cdot u \circ x\right), \quad u \in \mathbb{U}_{n} .
$$

\section{Definition 4.6}

Let $J \in \operatorname{Hol}\left(\mathbb{B}^{n}, \mathbb{C}^{n}\right)^{*}$ and $T>0$. Suppose that $u^{*} \in \mathcal{U}_{n}$. Then we define for each $t \geq 0$,

$$
L_{t} \in \operatorname{Hol}\left(\mathbb{B}^{n}, \mathbb{C}^{n}\right)^{*}, \quad L_{t}(u):=J\left(D x_{u^{*}}(T) \cdot\left(D x_{u^{*}}(t)\right)^{-1} \cdot u \circ x_{u^{*}}(t)\right) .
$$

Now we can state the analogue of Theorem 4.2 for the Loewner equation and general linear functionals $J \in \operatorname{Hol}\left(\mathbb{B}^{n}, \mathbb{C}^{n}\right)^{*}$.

Theorem 4.7 (Pontryagin Maximum Principle for the Loewner equation)

Let $J \in \operatorname{Hol}\left(\mathbb{B}^{n}, \mathbb{C}^{n}\right)^{*}$. Suppose that $u^{*}: \mathbb{R}_{0}^{+} \rightarrow \mathbb{U}_{n}$ is an optimal control for $J: \operatorname{Hol}\left(\mathbb{B}^{n}, \mathbb{C}^{n}\right) \rightarrow \mathbb{C}$ on $\mathbb{S}_{n}$ and time $T>0$. Then

$$
\operatorname{Re} L_{t}\left(u^{*}(t)\right)=\max _{u \in \mathbb{U}_{n}} \operatorname{Re} L_{t}(u) \quad \text { for a.e. } t \in[0, T] .
$$

It is now a short step to a statement of the Pontryagin Maximum Principle for the class $S_{n}^{0}$ and coefficient functionals $J_{\alpha}$.

Theorem 4.8 (Pontryagin Maximum Principle for the class $\mathcal{S}_{n}^{0}$ )

Let $\alpha \in \mathbb{N}_{0}^{N}$ with $|\alpha| \geq 2$ and let $F \in \mathcal{S}_{n}^{0}$ be an extremal mapping for $\operatorname{Re} J_{\alpha}$ over $\mathcal{S}_{n}^{0}$. Suppose that $G$ is a Herglotz vector field in the class $\mathbb{U}_{n}$ such that $F=f^{G}$. Denote by $\varphi_{t}: \mathbb{R}_{0}^{+} \rightarrow \mathbb{S}_{n}$ the solution to the Loewner equation (2.1). For each $t \geq 0$ define

$$
L_{t} \in \operatorname{Hol}\left(\mathbb{B}^{n}, \mathbb{C}^{n}\right)^{*}, \quad h \mapsto L_{t}(h):=J_{\alpha}\left(D F \cdot\left[D \varphi_{t}\right]^{-1} \cdot h\left(\varphi_{t}\right)\right) .
$$

Then for a.e. $t \geq 0$,

$$
\operatorname{Re} L_{t}(G(\cdot, t))=\max _{h \in \mathbb{U}_{n}} \operatorname{Re} L_{t}(h) .
$$

See [33] for a rigorous proof of Theorem 4.8.

\section{Conclusion 4.9}

Let $\alpha \in \mathbb{N}_{0}^{N}$ with $|\alpha| \geq 2$ and let $F \in S_{n}^{0}$. Suppose that $G$ is a Herglotz vector field in the class $\mathbb{U}_{n}$ such that $F=f^{G}$. Then the condition that

$$
G(\cdot, t) \text { maximizes } \operatorname{Re} L_{t} \text { over } \mathbb{U}_{n} \text { for a.e. } t \geq 0
$$

is necessary for $F$ being extremal for $\operatorname{Re} J_{\alpha}$ over $\mathcal{S}_{n}^{0}$. 


\section{The Schiffer differential equation and Pontryagin's maximum principle}

We now briefly describe the intimate relation between Schiffer's differential equation and Pontryagin's Maximum Principle for the case $\mathcal{S}_{1}^{0}=\mathcal{S}$. For the sake of simplicity, we restrict again to the case of the $N$-th coefficient functional $J_{N}: S \rightarrow \mathbb{C}, J_{N}(f):=$ $f^{(N)}(0) / N !$.

Theorem 5.1

Let $F \in \mathcal{S}$ and suppose that $G$ is a Herglotz vector field in the class $\mathbb{U}_{1}$ such that $F=f^{G}$. Denote by $\varphi_{t}: \mathbb{R}_{0}^{+} \rightarrow \mathbb{S}_{1}$ the solution to the Loewner equation (2.1). For each $t \geq 0$ define

$$
L_{t} \in \operatorname{Hol}(\mathbb{D}, \mathbb{C}), \quad h \mapsto L_{t}(h):=J_{N}\left(F^{\prime} \cdot\left(\varphi_{t}^{\prime}\right)^{-1} \cdot h\left(\varphi_{t}\right)\right)
$$

Then the following conditions are equivalent.

(a) F ist a solution of the Schiffer differential equation (1.2), that is,

$$
\left[\frac{z F^{\prime}(z)}{F(z)}\right]^{2} P_{N}\left(\frac{1}{F(z)}\right)=R_{N}(z),
$$

with $P_{N}$ resp. $R_{N}$ defined by (1.1) resp. (1.3), and the positivity condition (1.4) holds.

(b) For a.e. $t \geq 0$,

$$
\max _{h \in \mathbb{U}_{1}} \operatorname{Re} L_{t}(h)=\operatorname{Re} L_{t}(G(\cdot, t)) .
$$

Remark 5.2 (Schiffer's equation = Pontryagin's Maximum Principle)

Note that under the assumption that $F$ is extremal for the functional $J_{N}$ over $\mathcal{S}$ we have:

(i) Condition (a) = Conclusion of Schiffer's Theorem 1.2,

(ii) Condition ( $\mathrm{b})$ Conclusion of Pontryagin's Maximum Principle (Theorem 4.8 for the case $n=1$ ).

Hence Theorem 5.1 says that the two necessary conditions for $F$ being extremal for the functional $J_{N}$ over $\mathcal{S}$ provided by Schiffer's theorem (Condition (a)) and by Pontryagin's Maximum Principle (Condition (b)) are in fact equivalent.

A few more words are in order. 


\section{Remark 5.3}

The implication (a) $\Longrightarrow$ (b) of Theorem 5.1 can in fact be traced back to the work of Schaeffer, Schiffer and Spencer, see [37, 34, 35]. Consequently, a version of Pontryagin's Maximum Principle for a certain control system, namely the Loewner equation for $n=1$, has been known more than a decade before the discovery of the maximum principle by Pontryagin and his coauthors in 1956. In fact, Leung [23] speaks in this regard of the Schiffer-Pontryagin Maximum Principle. The converse implication (b) $\Longrightarrow$ (a) seems to lie deeper, see [11, 26, 32].

Conclusion 5.4 (Extending Schiffer's theorem to higher dimensions) Theorem 4.8 generalizes Schiffer's Theorem 1.2 from the class $\mathcal{S}=\mathcal{S}_{1}^{0}$ to any of the classes $S_{n}^{0}$.

\section{Teichmüller's coefficient theorem without quadratic differen- tials}

Using Theorem 5.1 it is now easy to give a formulation of Teichmüller's Coefficient Theorem solely in terms of the Loewner differential equation.

Theorem 6.1

Let $F \in \mathcal{S}$ and suppose that $G$ is a Herglotz vector field in the class $\mathbb{U}_{1}$ such that $F=f^{G}$. Denote by $\varphi_{t}: \mathbb{R}_{0}^{+} \rightarrow \mathbb{S}_{1}$ the solution to the Loewner equation (2.1). For each $t \geq 0$ define $L_{t} \in \operatorname{Hol}(\mathbb{D}, \mathbb{C})$ by (5.1). If for a.e. $t \geq 0$,

$$
\max _{h \in \mathbb{U}_{1}} \operatorname{Re} L_{t}(h)=\operatorname{Re} L_{t}(G(\cdot, t))
$$

then

$$
\operatorname{Re} J_{N}(f) \leq \operatorname{Re} J_{N}(F)
$$

for any

$$
f \in \mathcal{S}\left(A_{2}, \ldots, A_{N-1}\right) .
$$

Proof. By Theorem 5.1 and Remark 1.3 (d) this exactly is Teichmüller's Coefficient Theorem 1.4.

Note that in view of Pontryagin's Maximum Principle, condition (6.1) is necessary for $F \in \mathcal{S}$ being an extremal function for $J_{N}$ over $\mathcal{S}$. Theorem 6.1 simply says that this condition is also sufficient for extremality under a suitable side condition.

Conclusion 6.2

Let $F(z)=z+\sum_{k=2}^{\infty} A_{k} z^{k} \in \mathcal{S}$ and suppose that $G$ is a Herglotz vector field in the class $\mathbb{U}_{1}$ such that $F=f^{G}$. For each $t \geq 0$ define $L_{t} \in \operatorname{Hol}(\mathbb{D}, \mathbb{C})$ by $(5.1)$. Then the condition that 


$$
G(\cdot, t) \text { maximizes } \operatorname{Re} L_{t} \text { over } \mathbb{U}_{1} \text { for a.e. } t \geq 0
$$

is

(a) necessary for $F$ being extremal for $\operatorname{Re} J_{N}$ over $\mathcal{S}$, and

(b) sufficient for $F$ being extremal for $\operatorname{Re} J_{N}$ over $S\left(A_{2}, \ldots, A_{N-1}\right)$.

\section{Problem 6.3}

Find a proof of Theorem 6.1 using only the Loewner differential equation. We note that the standard method in control theory for obtaining sufficient conditions for optimal control functions makes use of Bellman functions. See [30] for some application of Bellman functions to the Loewner equation.

\section{Problem 6.4}

Let $\alpha \in \mathbb{N}_{0}^{N}$ be a multi-index with $|\alpha| \geq 2, F \in \mathcal{S}_{n}^{0}$ and suppose that $G$ is a Herglotz vector field in the class $\mathbb{U}_{n}$ such that $F=f^{G}$. Denote by $\varphi_{t}: \mathbb{R}_{0}^{+} \rightarrow \mathbb{S}_{n}$ the solution to the Loewner equation (2.1). For each $t \geq 0$ define $L_{t} \in \operatorname{Hol}\left(\mathbb{B}^{n}, \mathbb{C}^{n}\right)$ by (4.7). Assume that for a.e. $t \geq 0$,

$$
\max _{h \in \mathbb{U}_{n}} \operatorname{Re} L_{t}(h)=\operatorname{Re} L_{t}(G(\cdot, t)) .
$$

Is there a subset $\mathcal{S}_{\alpha}$ of $\mathcal{S}_{n}^{0}$ such that

$$
\operatorname{Re} J_{\alpha}(f) \leq \operatorname{Re} J_{\alpha}(F)
$$

for any $f \in \mathcal{S}_{\alpha}$ ? In other words, is the necessary condition for $F \in \mathcal{S}_{n}^{0}$ being an extremal function for $J_{\alpha}$ over $S_{n}^{0}$ provided by Pontryagin's Maximum Principle also sufficient under a suitable side condition? For $n=1$ the answer is "Yes" by Theorem 6.1. which we have seen is equivalent to Teichmüller's Coefficient Theorem 1.4. An affirmative answer for $n>1$ would therefore provide an extension of Teichmüller's Coefficient Theorem to higher dimensions.

\section{References}

[1] I. Aleksandrov. Parametric continuations in the theory of univalent functions. (Parametricheskie prodolzhenya teorii odnolistnykh funktsij). Moskva: "Nauka". 343 p., 1976.

[2] V. G. Boltyanskir, R. V. Gamkrelidze, and L. S. Pontryagin. On the theory of optimal processes. Dokl. Akad. Nauk SSSR (N.S.), 110:7-10, 1956. 
[3] F. Bracci, M. D. Contreras, S. Díaz-Madrigal, and A. Vasil'ev. Classical and stochastic Löwner-Kufarev equations. In Harmonic and complex analysis and its applications, pages 39-134. Cham: Birkhäuser/Springer, 2014.

[4] F. Bracci, I. Graham, H. Hamada, and G. Kohr. Variation of Loewner chains, extreme and support points in the class $S^{0}$ in higher dimensions. Constr. Approx., 43(2):231-251, 2016.

[5] F. Bracci and O. Roth. Support points and the bieberbach conjecture in higher dimension.

[6] R. Caccioppoli. Sui funzionali lineari nel campo delle funzioni analitiche. Atti Accad. Naz. Lincei, Rend., VI. Ser., 13:263-266, 1931.

[7] L. de Branges. A proof of the Bieberbach conjecture. Acta Math., 154:137-152, 1985.

[8] P. L. Duren. Univalent functions, volume 259 of Grundlehren der Mathematischen Wissenschaften [Fundamental Principles of Mathematical Sciences]. Springer-Verlag, New York, 1983.

[9] S. Friedland and M. Schiffer. Global results in control theory with applications to univalent functions. Bull. Amer. Math. Soc., 82(6):913-915, 1976.

[10] S. Friedland and M. Schiffer. On coefficient regions of univalent functions. J. Analyse Math., 31:125-168, 1977.

[11] G. S. Goodman. UNIVALENT FUNCTIONS AND OPTIMAL CONTROL. ProQuest LLC, Ann Arbor, MI, 1967. Thesis (Ph.D.)-Stanford University.

[12] I. Graham, H. Hamada, and G. Kohr. Parametric representation of univalent mappings in several complex variables. Canad. J. Math., 54(2):324-351, 2002.

[13] I. Graham, H. Hamada, G. Kohr, and M. Kohr. Parametric representation and asymptotic starlikeness in $\mathbb{C}^{n}$. Proc. Amer. Math. Soc., 136(11):3963-3973, 2008.

[14] I. Graham, H. Hamada, G. Kohr, and M. Kohr. Extreme points, support points and the Loewner variation in several complex variables. Sci. China Math., 55(7):1353-1366, 2012.

[15] I. Graham, H. Hamada, G. Kohr, and M. Kohr. Extremal properties associated with univalent subordination chains in $\mathbb{C}^{n}$. Math. Ann., 359(1-2):61-99, 2014.

[16] I. Graham and G. Kohr. Geometric function theory in one and higher dimensions, volume 255 of Monographs and Textbooks in Pure and Applied Mathematics. Marcel Dekker, Inc., New York, 2003. 
[17] A. Grothendieck. Sur certains espaces de fonctions holomorphes. i. J. Reine Angew. Math., 192:35-64, 1953.

[18] A. Grothendieck. Sur certains espaces de fonctions holomorphes. ii. J. Reine Angew. Math., 192:77-95, 1953.

[19] R. S. Hamilton. The inverse function theorem of Nash and Moser. Bull. Amer. Math. Soc. (N.S.), 7(1):65-222, 1982.

[20] J. A. Jenkins. Univalent functions and conformal mapping. Ergebnisse der Mathematik und ihrer Grenzgebiete. Neue Folge, Heft 18. Reihe: Moderne Funktionentheorie. Springer-Verlag, Berlin-Göttingen-Heidelberg, 1958.

[21] V. Jurdjevic. Geometric control theory, volume 52 of Cambridge Studies in Advanced Mathematics. Cambridge University Press, Cambridge, 1997.

[22] J. Koch and S. Schleißinger. Value ranges of univalent self-mappings of the unit disc. J. Math. Anal. Appl., 433(2):1772-1789, 2016.

[23] Y. J. Leung. Notes on Loewner differential equations. In Topics in complex analysis (Fairfield, Conn., 1983), volume 38 of Contemp. Math., pages 1-11. Amer. Math. Soc., Providence, RI, 1985.

[24] C. Pommerenke. Über die Subordination analytischer Funktionen. J. Reine Angew. Math., 218:159-173, 1965.

[25] C. Pommerenke. Univalent functions. Vandenhoeck \& Ruprecht, Göttingen, 1975. With a chapter on quadratic differentials by Gerd Jensen, Studia Mathematica/Mathematische Lehrbücher, Band XXV.

[26] V. Popov. L. S. Pontryagin's maximum principle in the theory of univalent functions. Sov. Math., Dokl., 10:1161-1164, 1969.

[27] D. Prokhorov. The method of optimal control in an extremal problem on a class of univalent functions. Sov. Math., Dokl., 29:301-303, 1984.

[28] D. Prokhorov and K. Samsonova. Value range of solutions to the chordal Loewner equation. J. Math. Anal. Appl., 428(2):910-919, 2015.

[29] D. V. Prokhorov. Sets of values of systems of functionals in classes of univalent functions. Mat. Sb., 181(12):1659-1677, 1990.

[30] D. V. Prokhorov. Reachable set methods in extremal problems for univalent functions. Saratov University Publishing House, Saratov, 1993. 
[31] D. V. Prokhorov. Bounded univalent functions. In Handbook of complex analysis: geometric function theory, Vol. 1, pages 207-228. North-Holland, Amsterdam, 2002.

[32] O. Roth. Pontryagin's maximum principle in geometric function theory. Complex Variables Theory Appl., 41(4):391-426, 2000.

[33] O. Roth. Pontryagin's maximum principle for the Loewner equation in higher dimensions. Canad. J. Math., 67(4):942-960, 2015.

[34] A. C. Schaeffer, M. Schiffer, and D. C. Spencer. The coefficient regions of schlicht functions. Duke Math. J., 16:493-527, 1949.

[35] A. C. Schaeffer and D. C. Spencer. Coefficient Regions for Schlicht Functions. American Mathematical Society Colloquium Publications, Vol. 35. American Mathematical Society, New York, N. Y., 1950. With a Chapter on the Region of the Derivative of a Schlicht Function by Arthur Grad.

[36] M. Schiffer. A method of variation within the family of simple functions. Proc. Lond. Math. Soc. (2), 44:432-449, 1938.

[37] M. Schiffer. Sur l'équation différentielle de M. Löwner. C. R. Acad. Sci. Paris, 221:369-371, 1945.

[38] E. Schippers. The power matrix, coadjoint action and quadratic differentials. J. Anal. Math., 98:249-277, 2006.

[39] S. Schleissinger. On support points of the class $S^{0}\left(B^{n}\right)$. Proc. Amer. Math. Soc., 142(11):3881-3887, 2014.

[40] K. Strebel. Quadratic differentials. Ergebnisse der Mathematik und ihrer Grenzgebiete. 3. Folge, Band 5. Berlin etc.: Springer-Verlag. XII, 184 p., 1984.

[41] O. Teichmüller. Ungleichungen zwischen den Koeffizienten schlichter Funktionen. Sitzungsber. Preuß. Akad. Wiss., Phys.-Math. Kl., 1938:363-375, 1938.

[42] O. Toeplitz. Die linearen vollkommenen Räume der Funktionentheorie. Comment. Math. Helv., 23:222-242, 1949.

[43] J. Zabczyk. Mathematical control theory: an introduction. Systems \& Control: Foundations \& Applications. Birkhäuser Boston, Inc., Boston, MA, 1992.

Oliver Roth

Department of Mathematics

University of Würzburg

Emil Fischer Straße 40 
97074 Würzburg

Germany

roth@mathematik.uni-wuerzburg.de 